\title{
Nonequilibrium molecular dynamics simulations of vibrational energy relaxation of HOD in $D_{2} O$
}

\author{
Alexander Kandratsenka, ${ }^{1,2, a)}$ Jörg Schroeder, ${ }^{2}$ Dirk Schwarzer, ${ }^{1}$ and \\ Vyacheslav S. Vikhrenko ${ }^{3}$ \\ ${ }_{1}^{1}$ Max-Planck-Institut für Biophysikalische Chemie, Am Faßberg 11, D-37077 Göttingen, Germany \\ ${ }^{2}$ Institut für Physikalische Chemie, Universität Göttingen, Tammannstrasse 6, D-37077 Göttingen, Germany \\ ${ }^{3}$ Department of Theoretical Mechanics, Belarussian State Technological University, Sverdlova Str. 13a, \\ 220006 Minsk, Belarus
}

(Received 14 January 2009; accepted 10 April 2009; published online 7 May 2009)

\begin{abstract}
Vibrational energy relaxation of HOD in deuterated water is investigated performing classical nonequilibrium molecular dynamics simulations. A flexible SPC/E model is employed to describe the intermolecular interactions and the intramolecular potential of the $\mathrm{D}_{2} \mathrm{O}$ solvent. A more accurate intramolecular potential is used for HOD. Our results for the OH stretch, OD stretch, and HOD bend vibrational relaxation times are 2.7, 0.9, and $0.57 \mathrm{ps}$, respectively. Exciting the $\mathrm{OH}$ stretching mode the main relaxation pathway involves a transition to the bending vibration. These results are in agreement with recent semiclassical Landau-Teller calculations. Contrary to this previous work, however, we observe a strong coupling of bending and $\mathrm{OH}$ stretching mode to the HOD rotation. As a result almost half of the total vibrational energy is transferred through the HOD rotation to the bath. At the same time the most efficient acceptor mode is the $\mathrm{D}_{2} \mathrm{O}$ rotation indicating the importance of resonant libration-to-libration energy transfer. We also find significant vibrational excitation of the $\mathrm{D}_{2} \mathrm{O}$ bending mode of the $\mathrm{D}_{2} \mathrm{O}$ solvent by $\mathrm{V}-\mathrm{V}$ energy transfer from the HOD bending mode. (C) 2009 American Institute of Physics. [DOI: 10.1063/1.3126781]
\end{abstract}

\section{INTRODUCTION}

Vibrational energy relaxation (VER) is of fundamental interest for reaction dynamics in a gaseous or liquid environment. The rate at which vibrational energy is redistributed in an excited molecule and exchanged with the surrounding medium depends on the specific interactions with the bath and can influence chemical reactivity and reaction pathways. As such, it is crucial to develop an in depth understanding of this process, in particular, for the liquid phase where a large amount of chemistry occurs. For small polyatomic molecules with only one quantum of vibrational excitation it is nowadays possible to explore VER mechanisms in great detail using a combination of ultrafast spectroscopy and theory. In the experiments a short infrared (IR) laser pulse is used to excite a particular vibration. ${ }^{1-3}$ The subsequent transfer of excess energy to other vibrational modes of the molecule and finally into the solvent can be probed by IR absorption, antiStokes Raman, or UV absorption spectroscopy, thereby monitoring time dependent populations of several vibrational states. Although these techniques deliver a substantial amount of information additional details of the relaxation cascade can be obtained by theoretical modeling usually based on molecular dynamics (MD) calculations.

In recent years liquid water played a unique role in understanding VER processes in a complex hydrogen bonded environment. In particular vibrational energy relaxation of the $\mathrm{OH}$ stretching mode of $\mathrm{HOD}$ in $\mathrm{D}_{2} \mathrm{O}$ has been the subject of numerous experimental ${ }^{4-16}$ and theoretical ${ }^{17-20}$ investiga-

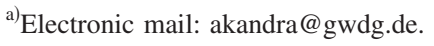

tions. This system was preferred to neat water because the $\mathrm{OH}$ stretch, $\nu_{\mathrm{OH}}$, of HOD is a local mode and spectrally well separated from vibrations of the solvent facilitating the observation of the VER process.

In the experiments femtosecond-IR-laser pulses around $3400 \mathrm{~cm}^{-1}$ were used to populate the $\nu_{\mathrm{OH}}=1$ state of the $\mathrm{OH}$ stretching vibration. The VER rate was determined by timeresolved monitoring of the excited state and ground state populations through absorption or Raman scattering of a delayed probe pulse. Dependent on the pump-probe detection scheme the lifetime $\tau_{\mathrm{OH}}$ of the $\mathrm{OH}$ excited state was found to be in the range of $0.5-1.0 \mathrm{ps}$. This broad range was attributed to a distribution of hydrogen bonded configurations of the HOD molecule, which on the timescale of VER appears to be partially inhomogeneous. As a result the relaxation is faster for a subensemble of strongly hydrogen-bonded $\mathrm{OH}-$ oscillators absorbing at the red edge of the steady state absorption spectrum as compared to a weakly hydrogenbonded subensemble excited at the blue edge. ${ }^{13}$ Above $400 \mathrm{~K}$ the dispersion of $\tau_{\mathrm{OH}}$ disappears because with increasing temperature the reorganization of local hydrogen-bonded configurations accelerates and, simultaneously, the $\mathrm{OH}$ stretch relaxation becomes slower as the hydrogen bond network is weakened. ${ }^{15,16}$ Interestingly, at these higher temperatures a linear relationship between the $\mathrm{OH}$ stretch relaxation rate and the static dielectric constant of water was found, which by extrapolation was used to determine the ensemble averaged lifetime of the first excited vibrational quantum state of $\nu_{\mathrm{OH}}$ to $\tau_{\mathrm{OH}}=0.63 \mathrm{ps}$ at ambient conditions. ${ }^{15}$

The important question concerning the energy relaxation path from $\mathrm{OH}$ stretch via solute modes into the bath was 
addressed experimentally by a time resolved IR pump/ Raman probe study of Deak et al. ${ }^{21}$ They found out that within 1 ps after $\nu_{\mathrm{OH}}$ excitation not only the bending vibration $\delta_{\mathrm{HOD}}$ of HOD but also bending $\left(\delta_{\mathrm{DOD}}\right)$, symmetric $\left(\nu_{\mathrm{SS}}\right)$ and asymmetric $\left(\nu_{\mathrm{as}}\right)$ stretching vibrations of the solvent $\mathrm{D}_{2} \mathrm{O}$ are transiently excited. Bakker and co-workers ${ }^{8,12}$ made use of a vibrational predissociation mechanism ${ }^{22}$ to interpret the unusual temperature dependence of the $\mathrm{OH}$ stretch relaxation time. According to this model the accepting mode of the $\mathrm{OH}$ stretch excess energy is the hydrogen bond $\mathrm{DOH} \cdots \mathrm{OD}_{2}$, which subsequently breaks.

Comprehensive information on the relaxation pathways was obtained from theoretical calculations based on the Landau-Teller (LT) method. ${ }^{17,18,20,23}$ In this approach the solvent degrees of freedom are treated classically, while the quantum nature of the solute HOD enters through the transition moments of the molecular normal modes. From a MD simulation of a rigid HOD molecule in a bath of $\mathrm{D}_{2} \mathrm{O}$ molecules classical time correlation functions (TCF) are calculated from which the individual vibrational state-to-state rate constants of HOD are evaluated. The mechanistic picture emerging from these studies involves a rate-determining transfer from the excited $\nu_{\mathrm{OH}}$ to the first overtone of the HOD bend as the major relaxation channel. However, there are several drawbacks of this method. First of all a quantum correction factor $(\mathrm{QCF})$ has to be introduced to calculate the quantum rate constant from a classical TCF. Unfortunately, this problem has not been solved entirely and there are many QCFs available in the literature which can differ by orders of magnitude. Furthermore, the LT method is a perturbative approach. Some relaxation processes in the HOD molecule, however, are so fast that the weak-coupling assumption may not be justified. This is particularly the case for the bending vibration, which relaxes within a few hundred femtoseconds. Also, the LT approach does not allow to treat the solvent response upon $\nu_{\mathrm{OH}^{-}}$excitation or a possible $\mathrm{DOH} \cdot \mathrm{OD}_{2}$ vibrational predissociation.

An alternative to LT calculations are completely classical nonequilibrium MD (NEMD) simulations. $^{24-26}$ Of course, this method disregards completely the quantum nature of high frequency vibrations. On the other hand many properties of classical and quantum oscillators are known to show similar behavior. This fact has been exploited in numerous studies where NEMD calculations have been shown to give reliable results even for molecules with high frequency vibrations such as azulene, ${ }^{27,28} \mathrm{CH}_{2} \mathrm{I}_{2},{ }^{29} \mathrm{CH}_{3} \mathrm{NO}_{2},{ }^{30}$ $\mathrm{CO}_{2},{ }^{31,32}$ and large bichromophoric molecules. ${ }^{33}$ It may be noted, however, that there are cases where classical NEMD fails to describe VER properly. For $\mathrm{CHBr}_{3}$ the zero point energy had to be added to the excitation energy to reproduce the experimentally observed relaxation rate. ${ }^{34}$

Classical NEMD calculations do not rely on restrictions like weak solute-solvent interactions. Hence, e.g., strongly coupled solute-bath systems as well as the response of the solvent under nonequilibrium conditions can be studied. For VER of $\mathrm{CH}_{3} \mathrm{Cl}$ in water, NEMD and classical LT calculations were found to be in a good agreement. ${ }^{25}$

Based on NEMD one may elucidate VER pathways in great detail by analyzing the mode specific work $^{25,28,35}$ per- formed by the solute on the bath. In the present article this approach is used to investigate the relaxation mechanism of the excited $\mathrm{OH}$ stretching vibration of $\mathrm{HOD}$ in $\mathrm{D}_{2} \mathrm{O}$. We will show that many of the features found with the LT method are also captured by NEMD. In addition, new pathways are identified as, e.g., direct vibration-to-vibration (V-V) energy transfer of the HOD bend to the $\mathrm{D}_{2} \mathrm{O}$ bend vibration, which was suggested by time-resolved Raman experiments, ${ }^{21}$ but have not been recovered by previous theoretical calculations.

\section{THEORETICAL BACKGROUND}

\section{A. Separation of molecular motions}

To investigate the energy transfer routes we start with the expression for the solute energy

$$
E=K+U \text {, }
$$

where the intramolecular potential energy

$$
U=U_{1}+U_{2}+U_{n}
$$

is represented by a sum of terms depending on one $\left(U_{1}\right)$, two $\left(U_{2}\right)$, and more than two $\left(U_{n}\right)$ normal coordinates $Q_{\mu}$, namely,

$$
\begin{aligned}
U_{1}= & \sum_{\mu=1}^{s}\left(k_{\mu \mu} Q_{\mu}^{2}+k_{\mu \mu \mu} Q_{\mu}^{3}+k_{\mu \mu \mu \mu} Q_{\mu}^{4}+\cdots\right), \\
U_{2}= & \sum_{\mu<\nu}^{s}\left(k_{\mu \nu \nu} Q_{\mu} Q_{\nu}^{2}+k_{\mu \mu \nu \nu} Q_{\mu}^{2} Q_{\nu}^{2}+\cdots\right), \\
U_{n}= & \sum_{\mu<\nu<\gamma \ldots}^{s}\left(k_{\mu \nu \gamma} Q_{\mu} Q_{\nu} Q_{\gamma}+k_{\mu \nu \gamma \gamma} Q_{\mu} Q_{\nu} Q_{\gamma}^{2}\right. \\
& \left.+k_{\mu \nu \gamma \delta} Q_{\mu} Q_{\nu} Q_{\gamma} Q_{\delta}+\cdots\right) .
\end{aligned}
$$

Here $k_{\mu \nu} \ldots$ are force constants and $s$ is the total number of vibrational degrees of freedom. by $^{36-38}$

The kinetic energy of the solute molecule is given

$$
\begin{aligned}
K & =\frac{1}{2} \sum_{\mu=1}^{s} \dot{Q}_{\mu}^{2}+\frac{1}{2} \boldsymbol{\omega} \cdot \boldsymbol{I} \cdot \boldsymbol{\omega}+\boldsymbol{\omega} \cdot \sum_{\mu, \nu=1}^{s} \boldsymbol{\zeta}_{\mu \nu} Q_{\mu} \dot{Q}_{\nu} \\
& =K_{\mathrm{vibr}}+K_{\mathrm{rot}}+K_{\mathrm{cor}} .
\end{aligned}
$$

The first term of Eq. (4) represents the sum of vibrational normal mode kinetic energies, the second term accounts for rotational motion of the molecule ( $\boldsymbol{\omega}$ is the angular velocity of the rigid configuration associated with the equilibrium geometry of the molecule, $\boldsymbol{I}$ is the inertia tensor), and the last term describes Coriolis coupling between rotations and vibrations.

We used the Eckart frame $\mathrm{e}^{37-39}$ to separate vibrational and rotational motions. In this case $\mathrm{c}^{36,40}$

$$
\boldsymbol{I}=\boldsymbol{I}_{0}+\sum_{\mu} \boldsymbol{B}_{\mu} Q_{\mu}+\frac{1}{2} \sum_{\mu, \nu} \boldsymbol{D}_{\mu \nu} Q_{\mu} Q_{\nu},
$$




$$
\boldsymbol{I}_{0}=\sum_{a} m_{a}\left(a_{a}^{2} \boldsymbol{E}-\boldsymbol{a}_{a} \boldsymbol{a}_{a}\right)
$$

is the inertia tensor of the equilibrium configuration given by the nuclear position vectors $\boldsymbol{a}_{a}$ in the center of mass coordinate system, $m_{a}$ are atomic masses, $\boldsymbol{E}$ is the unit tensor, and

$$
\begin{aligned}
& \boldsymbol{B}_{\mu}=\sum_{a} m_{a}^{-1 / 2}\left[2\left(\boldsymbol{a}_{a} \cdot \boldsymbol{L}_{a \mu}\right) \boldsymbol{E}-\left(\boldsymbol{a}_{a} \boldsymbol{L}_{a \mu}+\boldsymbol{L}_{a \mu} \boldsymbol{a}_{a}\right)\right], \\
& \boldsymbol{D}_{\mu \nu}=\sum_{a}\left[2\left(\boldsymbol{L}_{a \mu} \cdot \boldsymbol{L}_{a \nu}\right) \boldsymbol{E}-\left(\boldsymbol{L}_{a \mu} \boldsymbol{L}_{a \nu}+\boldsymbol{L}_{a \nu} \boldsymbol{L}_{a \mu}\right)\right] .
\end{aligned}
$$

The Coriolis coupling coefficients

$$
\boldsymbol{\zeta}_{\mu \nu}=\sum_{a} \boldsymbol{L}_{a \mu} \times \boldsymbol{L}_{a \nu}
$$

are defined by the normal vectors $\boldsymbol{L}_{a \mu}$ that represent instantaneous atomic displacements from the equilibrium positions in terms of normal coordinates,

$$
\boldsymbol{r}_{a}=\boldsymbol{a}_{a}+m_{a}^{-1 / 2} \sum_{\mu} \boldsymbol{L}_{a \mu} Q_{\mu}
$$

Orthogonality of normal modes imply the following relations between these vectors ${ }^{36}$

$$
\begin{aligned}
& \sum_{a} \sqrt{m_{a}} \boldsymbol{L}_{a \mu}=0, \\
& \sum_{a} \sqrt{m_{a}} \boldsymbol{a}_{a} \times \boldsymbol{L}_{a \mu}=0, \\
& \sum_{a} \boldsymbol{L}_{a \mu} \cdot \boldsymbol{L}_{a \nu}=\delta_{\mu \nu} .
\end{aligned}
$$

\section{B. Equations of motion for vibrational and rotational degrees of freedom}

For a molecule the generalized momentum $P_{\mu}$ conjugate to the normal coordinate $Q_{\mu}$ is defined as

$$
P_{\mu}=\frac{\partial K}{\partial \dot{Q}_{\mu}}=\dot{Q}_{\mu}+\boldsymbol{\omega} \cdot \sum_{\nu} \zeta_{\nu \mu} Q_{\nu} .
$$

The Lagrange equations

$$
\dot{P}_{\mu}-\frac{\partial K}{\partial Q_{\mu}}=-\frac{\partial U}{\partial Q_{\mu}}-\frac{\partial V}{\partial Q_{\mu}}
$$

describe the evolution of normal coordinates, where $V$ represents the solute-bath interaction energy. Taking into account Eqs. (12) and (4), from Eq. (13) follows

$$
\begin{aligned}
\ddot{Q}_{\mu}= & F_{\mu}-2 \boldsymbol{\omega} \cdot \sum_{\nu} \zeta_{\nu \mu} \dot{Q}_{\nu}+\frac{1}{2} \boldsymbol{\omega} \cdot \frac{\partial \boldsymbol{I}}{\partial Q_{\mu}} \cdot \boldsymbol{\omega} \\
& +\dot{\boldsymbol{\omega}} \cdot \sum_{\nu} \zeta_{\nu \mu} Q_{\nu},
\end{aligned}
$$

where the first term

$$
F_{\mu}=F_{\mu}^{(e)}+F_{\mu}^{(i)}=-\frac{\partial U}{\partial Q_{\mu}}-\frac{\partial V}{\partial Q_{\mu}}
$$

is the sum of external and intramolecular forces acting on the $\mu$ th mode and the derivative $\partial \boldsymbol{I} / \partial Q_{\mu}$ is obtained from Eq. $(5)$,

$$
\frac{\partial \boldsymbol{I}}{\partial Q_{\mu}}=\boldsymbol{B}_{\mu}+\sum_{\nu} \boldsymbol{D}_{\mu \nu} Q_{\nu} .
$$

Equation (14) represents the equations of motion for the vibrational degrees of freedom.

The time evolution of rotational degrees of freedom is governed by the equation ${ }^{41}$

$$
\frac{d \boldsymbol{L}_{C}}{d t}=\boldsymbol{M}_{C},
$$

where $\boldsymbol{M}_{C}$ is the torque of the external forces exerted on the solute with respect to its center of mass $C$ and

$$
\boldsymbol{L}_{C}=\boldsymbol{I} \cdot \boldsymbol{\omega}+\sum_{\mu \nu} \boldsymbol{\zeta}_{\mu \nu} Q_{\mu} \dot{Q}_{\nu}
$$

is the total angular momentum of the solute where the last term describes the vibrational contribution to the angular momentum.

In the following the notation $d / d t$ is used for the time derivative in the laboratory frame, while a dot over a symbol designates the time derivative in the rotating Eckart bodyfixed frame. These derivatives satisfy the relation ${ }^{41,42}$

$$
\frac{d \boldsymbol{A}}{d t}=\dot{\boldsymbol{A}}+\boldsymbol{\omega} \times \boldsymbol{A},
$$

which holds for any $\boldsymbol{A}$. Note that the time derivative of a scalar function does not depend on the coordinate system used. Additionally, the time derivatives of the angular velocity are equal in both frames and the term $\boldsymbol{\omega} \cdot\left(d \boldsymbol{\zeta}_{\mu \nu} / d t\right)$ $=\boldsymbol{\omega} \cdot\left(\boldsymbol{\omega} \times \boldsymbol{\zeta}_{\mu \nu}\right)=0$ does not appear in Eq. (14) because vectors $\zeta_{\mu \nu}$ are constant in the body fixed frame.

Due to the antisymmetry of the Coriolis coupling coefficients (9) with respect to permutation of their subscripts the time derivative of Eq. (18) in the Eckart frame is

$$
\dot{\boldsymbol{L}}_{C}=\dot{\boldsymbol{I}} \cdot \boldsymbol{\omega}+\boldsymbol{I} \cdot \dot{\boldsymbol{\omega}}+\sum_{\mu \nu} \zeta_{\mu \nu} Q_{\mu} \ddot{Q}_{\nu}
$$

such that Eq. (17) takes the form

$$
\begin{aligned}
\boldsymbol{I} \cdot \dot{\boldsymbol{\omega}}= & \boldsymbol{M}_{C}-\dot{\boldsymbol{I}} \cdot \boldsymbol{\omega}-\boldsymbol{\omega} \times(\boldsymbol{I} \cdot \boldsymbol{\omega})-\boldsymbol{\omega} \times \sum_{\mu \nu} \zeta_{\mu \nu} Q_{\mu} \dot{Q}_{\nu} \\
& -\sum_{\mu \nu} \zeta_{\mu \nu} Q_{\mu} \ddot{Q}_{\nu} .
\end{aligned}
$$

Equation (21) describes the dynamics of the rotational degrees of freedom of the solute in the bath.

It turns out to be useful for manipulations with NEMD simulation results to decouple Eqs. (14) and (21) with respect to the accelerations substituting the former into the latter. After some algebra we arrive at the expression 


$$
\begin{aligned}
\boldsymbol{I}^{\prime} \cdot \dot{\boldsymbol{\omega}}= & \tilde{\boldsymbol{M}}_{C}-\dot{\boldsymbol{I}}^{\prime} \cdot \boldsymbol{\omega}-\boldsymbol{\omega} \times\left(\boldsymbol{I}^{\prime} \cdot \boldsymbol{\omega}\right)-\boldsymbol{\omega} \times \sum_{\mu \nu} \boldsymbol{\zeta}_{\mu \nu} Q_{\mu} \dot{Q}_{\nu} \\
& -\frac{1}{2} \sum_{\mu \nu} \boldsymbol{\zeta}_{\mu \nu} Q_{\mu} \boldsymbol{\omega} \cdot \frac{\partial \boldsymbol{I}^{\prime}}{\partial Q_{\nu}} \cdot \boldsymbol{\omega}
\end{aligned}
$$

where the modified inertia tensor ${ }^{36}$

$$
\begin{aligned}
& \boldsymbol{I}^{\prime}=\boldsymbol{I}-\sum_{\mu \nu \tau} \zeta_{\mu \nu} \zeta_{\tau \nu} Q_{\mu} Q_{\tau}=\boldsymbol{I}^{\prime \prime} \cdot \boldsymbol{I}_{0}^{-1} \cdot \boldsymbol{I}^{\prime \prime}, \\
& \boldsymbol{I}^{\prime \prime}=\boldsymbol{I}_{0}+\frac{1}{2} \sum_{\mu} \boldsymbol{B}_{\mu} Q_{\mu},
\end{aligned}
$$

and the modified torque of the external forces

$$
\tilde{\boldsymbol{M}}_{C}=\boldsymbol{I}^{\prime \prime} \cdot \boldsymbol{I}_{0}^{-1} \cdot \sum_{a} \boldsymbol{a}_{a} \times \boldsymbol{F}_{a}
$$

are used. Substitution of Eq. (22) solved with respect to $\dot{\boldsymbol{\omega}}$ into Eq. (14) yields the corresponding equation for $\ddot{Q}_{\mu}$.

\section{Time evolution of mode specific energies}

The equations of motion for the rotational and vibrational degrees of freedom allow us to calculate time derivatives of mode-specific contributions to the molecular energy. To this end it is useful to include the anharmonic contributions of Eq. (3) into the normal mode energies:

$$
E_{\mu}=\frac{1}{2} \dot{Q}_{\mu}^{2}+U_{1 \mu}
$$

Its time derivative calculated from Eq. (14),

$$
\begin{aligned}
\frac{d E_{\mu}}{d t}= & F_{\mu}^{(e)} \dot{Q}_{\mu}-\frac{\partial U_{2}}{\partial Q_{\mu}} \dot{Q}_{\mu}-\frac{\partial U_{n}}{\partial Q_{\mu}} \dot{Q}_{\mu}-2 \boldsymbol{\omega} \cdot \sum_{\nu} \boldsymbol{\zeta}_{\mu \nu} \dot{Q}_{\nu} \dot{Q}_{\mu} \\
& +\frac{1}{2} \boldsymbol{\omega} \cdot\left(\frac{\partial \boldsymbol{I}}{\partial Q_{\mu}} \dot{Q}_{\mu}\right) \cdot \boldsymbol{\omega}+\dot{\boldsymbol{\omega}} \cdot \sum_{\nu} \boldsymbol{\zeta}_{\mu \nu} Q_{\nu} \dot{Q}_{\mu},
\end{aligned}
$$

contains terms with well-defined physical meaning. $F_{\mu}^{(e)}$ is the projection of the external force on the $\mu$ th vibrational coordinate. Thus, the first term on the right-hand side (r.h.s.) of Eq. (25) describes the energy exchange between a vibrational mode $\mu$ and the bath.

The second term,

$$
\frac{\partial U_{2}}{\partial Q_{\mu}}=\sum_{\nu \neq \mu}\left(k_{\mu \nu \nu} Q_{\nu}^{2}+k_{\mu \nu \nu \nu} Q_{\nu}^{3}+\ldots+2 k_{\mu \mu \nu} Q_{\mu} Q_{\nu}+\cdots\right),
$$

arises from binary couplings of mode $\mu$ to other normal modes $\nu$, and hence accounts for intramolecular energy transfer between two particular vibrational degrees of freedom. The third term describes multimode intramolecular energy transfer and cannot be decomposed into binary contributions.

The last three terms on the r.h.s. of Eq. (25) show the importance of kinematic nonlinearities. As clarified in Appendix B the first of them takes into account Coriolis coupling and describes energy transfer from mode $\mu$ to mode $\nu$ mediated by the rotation of the molecule. The second term includes energy exchange between vibrations and rotations due to centrifugal coupling. The partial derivative $\partial \boldsymbol{I} / \partial Q_{\mu}$ comprises terms depending on $Q_{\mu}$ and $Q_{\nu}$ [see Eq. (16)]. Therefore, this energy flow can be divided into a binary part (from mode $\mu$ to rotation) and nonbinary component coupling two vibrational modes $\mu$ and $\nu$ to the rotations. The last term in Eq. (25) is governed by the angular acceleration $\dot{\boldsymbol{\omega}}$ and describes energy transfer from mode $\mu$ to rotation mediated by a mode $\nu$. This so-called Euler contribution ${ }^{42}$ is evidently of nonbinary character.

All the terms on the right hand side of Eq. (25) can be considered as power contributions delivered by the underlying forces. Their time integrals yield corresponding work contributions that give full information about the intra- and intermolecular energy exchange during VER. In accordance with Eq. (25) the change of energy in the mode $\mu$,

$$
\Delta E_{\mu}=W_{\mu}^{(\mathrm{ext})}+W_{\mu}^{(\mathrm{int})}+W_{\mu}^{(\mathrm{cf})}+W_{\mu}^{(\mathrm{cor})}+W_{\mu}^{(\mathrm{eul})},
$$

is caused by the sum of work contributions performed by the other degrees of freedom via external, internal, centrifugal, Coriolis, and Euler forces.

\section{Solute-solvent intermode energy exchange}

In the present subsection the work $W_{\mu}^{(\mathrm{ext})}$ done by external forces on the coordinate $\mu$ is decomposed into contributions from different vibrational degrees of freedom of the solvent. In MD simulations intermolecular interactions are calculated from the sum of atomic pair potentials

$$
V_{i j}=\sum_{a b} V_{i a j b}
$$

where $V_{i a j b}$ is the interaction potential between atom $a$ of molecule $i$ and atom $b$ of molecule $j$.

For analyzing mode-to-mode intermolecular energy transfer the potential has to be expanded in terms of the normal vibrational coordinates $Q_{i \tau}$ and $Q_{j \mu}$ of the two molecules:

$$
\begin{aligned}
V_{i j}= & V_{i j}^{0}-\sum_{\tau} a_{i \tau}^{(i j)} Q_{i \tau}-\sum_{\mu} a_{j \mu}^{(i j)} Q_{j \mu}-\frac{1}{2} \sum_{\tau \tau^{\prime}} b_{i \tau i \tau^{\prime}}^{(i j)} Q_{i \tau} Q_{i \tau^{\prime}} \\
& -\frac{1}{2} \sum_{\mu \mu^{\prime}} b_{j \mu j \mu^{\prime}}^{(i j)} Q_{j \mu} Q_{j \mu^{\prime}}-\sum_{\tau \mu} b_{i \tau j \mu}^{(i j)} Q_{i \tau} Q_{j \mu}+\Delta V_{i j},
\end{aligned}
$$

where $\Delta V_{i j}$ includes the higher order expansion terms. The expansion coefficients depend on the particular arrangement of molecules $i$ and $j$ at a given instant of time and are defined by

$$
a_{i \tau}^{(i j)}=-\left.\frac{\partial V_{i j}}{\partial Q_{i \tau}}\right|_{Q=0}, \quad b_{i \tau j \mu}^{(i j)}=-\left.\frac{\partial^{2} V_{i j}}{\partial Q_{i \tau} Q_{j \mu}}\right|_{Q=0},
$$

where subscript $Q=0$ indicates that after taking the derivative all the vibrational coordinates have to be set to their equilibrium values.

Equation (29) leads to the corresponding expansion for 
the projected intermolecular force $F_{\tau}^{(i j)}$ of molecule $j$ exerted on normal mode $\tau$ th of molecule $i$

$$
F_{\tau}^{(i j)}=-\frac{\partial V^{(i j)}}{\partial Q_{i \tau}}=a_{i \tau}^{(i j)}+\sum_{\tau^{\prime}} b_{i \pi i \tau^{\prime}}^{(i j)} Q_{i \tau^{\prime}}+\sum_{\mu} b_{i \tau j \mu}^{(i j)} Q_{j \mu}+\Delta F_{\tau}^{i j}
$$

and for the power $N_{\tau}^{(i j)}$ of the normal force

$$
\begin{aligned}
N_{\tau}^{(i j)}= & F_{\tau}^{(i j)} \dot{Q}_{i \tau}=a_{i \tau}^{(i j)} \dot{Q}_{i \tau}+b_{i \pi i \tau}^{(i j)} Q_{i \tau} \dot{Q}_{i \tau}+\sum_{\tau^{\prime} \neq \tau} b_{i \pi i \tau^{\prime}}^{(i j)} Q_{i \tau^{\prime}} \dot{Q}_{i \tau} \\
& +\sum_{\mu} b_{i \tau j \mu}^{(i j)} Q_{j \mu} \dot{Q}_{i \tau}+\Delta N_{\tau}^{i j} .
\end{aligned}
$$

Here the first three terms on the r.h.s. describe energy transfer from vibrational mode $\tau$ of molecule $i$ to translations and rotations of molecule $j$. Their translational and rotational contributions cannot be clearly separated. The forth term is responsible for direct $\mathrm{V}-\mathrm{V}$ transfer between mode $\tau$ of molecule $i$ and mode $\mu$ of molecule $j$.

The partial derivatives of the intermolecular potentials entering Eqs. (30) can be expressed by the equilibrium positions of the molecules $i$ and $j$ at a particular time $t$,

$$
\begin{aligned}
& \frac{\partial V_{(i a j b)}}{\partial Q_{i \tau}}=m_{i a}^{-1 / 2} \phi_{1}\left(\boldsymbol{r}_{i a j b}\right) \cdot \boldsymbol{L}_{i a \tau}, \\
& \frac{\partial^{2} V_{(i a j b)}}{\partial Q_{i \tau} \partial Q_{j \mu}}=\left(m_{i a} m_{j b}\right)^{-1 / 2} \boldsymbol{L}_{i a \tau} \cdot \boldsymbol{\phi}_{2}\left(\boldsymbol{r}_{i a j b}\right) \cdot \boldsymbol{L}_{j b \mu},
\end{aligned}
$$

where the definition (10) was taken into account and the notations

$$
\begin{aligned}
& \phi_{1}(\boldsymbol{r})=\frac{\partial V(r)}{\partial \boldsymbol{r}}=\Phi_{1}(r) \boldsymbol{r}, \\
& \phi_{2}(\boldsymbol{r})=\frac{\partial^{2} V(r)}{\partial \boldsymbol{r} \partial \boldsymbol{r}}=\Phi_{2}(r) \boldsymbol{r} \boldsymbol{r}+\Phi_{1}(r) \boldsymbol{E}, \\
& \Phi_{1}(r)=\frac{1}{r} \frac{\partial V(r)}{\partial r}, \quad \Phi_{2}(r)=\frac{\partial^{2} V(r)}{\partial r^{2}}-\Phi_{1}(r)
\end{aligned}
$$

were introduced.

Thus, for a given configuration the expansion coefficients are calculated by resetting the molecular geometries to their equilibrium values using the Eckart frame,

$$
\begin{aligned}
& a_{i \tau}^{(i j)}=\sum_{a, b} m_{i a}^{-1 / 2} \boldsymbol{L}_{i a \tau} \cdot \phi_{1}\left(\boldsymbol{a}_{i a j b}\right), \\
& b_{i \pi i \tau^{\prime}}^{(i j)}=-\sum_{a, b} m_{i a}^{-1} \boldsymbol{L}_{i a \tau} \cdot \phi_{2}\left(\boldsymbol{a}_{i a j b}\right) \cdot \boldsymbol{L}_{i a \tau^{\prime}}, \\
& b_{i \pi j \mu}^{(i j)}=-\sum_{a, b}\left(m_{i a} m_{j b}\right)^{-1 / 2} \boldsymbol{L}_{i a \tau} \cdot \phi_{2}\left(\boldsymbol{a}_{i a j b}\right) \cdot \boldsymbol{L}_{j b \mu},
\end{aligned}
$$

where $\boldsymbol{a}_{i a j b}$ is a vector joining the equilibrium position of atom $a$ of molecule $i$ with that of atom $b$ of molecule $j$ [see Eq. (10)].

\section{MOLECULAR DYNAMICS SIMULATION DETAILS}

NEMD simulations of a single HOD molecule immersed in a bath of $107 \mathrm{D}_{2} \mathrm{O}$ solvent molecules were performed by applying standard simulation techniques. ${ }^{43}$ Similar to the LT calculations of Ref. 17 a flexible SPC/E model was employed to describe intermolecular water interactions and the intramolecular $\mathrm{D}_{2} \mathrm{O}$ potential. For the latter, however, in contrast with Ref. 17 different force constants were applied, ${ }^{44}$ which reproduce better the experimental vibrational frequencies of $\mathrm{D}_{2} \mathrm{O}$.

For HOD the intramolecular force field of Sceats and Rice $^{45}$ was used

$$
\begin{aligned}
U= & \frac{1}{2} k_{r r}\left(\Delta r_{1}^{2}+\Delta r_{2}^{2}\right)+\frac{1}{2} k_{\alpha \alpha} r_{\mathrm{eq}}^{2} \Delta \alpha^{2} \\
& +k_{r \alpha} r_{\mathrm{eq}} \Delta \alpha\left(\Delta r_{1}+\Delta r_{2}\right)+k_{r r} \Delta r_{1} \Delta r_{2} \\
& +\frac{k_{r r r}}{r_{\mathrm{eq}}}\left(\Delta r_{1}^{3}+\Delta r_{2}^{3}\right)+\frac{k_{r r r r}}{r_{\mathrm{eq}}^{2}}\left(\Delta r_{1}^{4}+\Delta r_{2}^{4}\right),
\end{aligned}
$$

where $r_{1}, r_{2}$, and $\alpha$ refer to the $\mathrm{OH}$ and $\mathrm{OD}$ bond lengths and to the HOD angle, respectively. The force constants [in $\left.\mathrm{kJ} /\left(\mathrm{mol} \AA^{2}\right)\right]$ are

$$
\begin{aligned}
& k_{r r}=5085.8(1-\eta)^{2}, \quad k_{r r^{\prime}}=-79.49+1276.1 \eta, \\
& k_{\alpha \alpha}=482.25, \quad k_{r \alpha}=283.281, \\
& k_{r r r}=5514.9, \quad k_{r r r r}=8334.0 .
\end{aligned}
$$

The parameter $\eta$ accounts for the decrease of the harmonic stretching frequency $\omega_{r}$ from its gas-phase value $\omega_{r}^{\text {(gas) }}$,

$$
\omega_{r}(\eta)=\omega_{r}^{(\text {gas })}(1-\eta) .
$$

For the isolated HOD molecule $\eta=0.056$ gives the correct liquid phase frequency. ${ }^{17,45}$ However, when HOD is surrounded by water molecules, solvent-bath interactions lead to an additional redshift of $\omega_{r}$. Consequently, $\eta$ had to be decreased to 0.026 to match the actual liquid phase stretching frequency in our MD simulations.

To analyze the mode specific energy transfer it is convenient to transform Eq. (36) into a dimensionless normal coordinate representation ${ }^{46}$

$$
\begin{aligned}
\frac{V}{h c}= & \frac{1}{2} \sum_{\tau} \widetilde{\nu}_{\tau} q_{\tau}^{2}+\sum_{\tau \leq \mu \leq \sigma} k_{\tau \mu \sigma} q_{\tau} q_{\mu} q_{\sigma} \\
& +\sum_{\tau \leq \mu \leq \sigma \leq \nu} k_{\tau \mu \sigma \nu} q_{\tau} q_{\mu} q_{\sigma} q_{\nu} .
\end{aligned}
$$

The force constants are given in Table I.

The decomposition of the intramolecular potential into normal modes up to the fourth order [Eq. (38)] was implemented for our MD calculations. As the Eckart frame is utilized to separate rotations and vibrations, a special procedure described in Appendix A was developed to preserve the rotational invariance of intramolecular HOD interactions.

MD simulations were performed by applying the modified Beeman algorithm ${ }^{47}$ with an integration time step of 0.04 fs. Electrostatic forces were handled with the Ewald summation technique. ${ }^{43}$ After an equilibration period of 
TABLE I. Force constants (in units of $\mathrm{cm}^{-1}$ ) for the HOD intramolecular potential.

\begin{tabular}{lcc}
\hline \hline$\widetilde{\nu}_{1}=2751.9$ & $\widetilde{\nu}_{2}=1440.1$ & $\widetilde{\nu}_{3}=3787.4$ \\
$k_{111}=-266.1$ & $k_{112}=-125.0$ & $k_{113}=40.5$ \\
$k_{122}=31.8$ & $k_{123}=38.3$ & $k_{133}=-57.0$ \\
$k_{222}=-16.7$ & $k_{223}=220.6$ & $k_{233}=166.0$ \\
& $k_{333}=-433.4$ & \\
$k_{1111}=34.2$ & $k_{1133}=1.22$ & $k_{2222}=7.8$ \\
$k_{1112}=25.7$ & $k_{1222}=-5.0$ & $k_{2223}=-25.8$ \\
$k_{1113}=-7.4$ & $k_{1223}=-6.3$ & $k_{2233}=-126.4$ \\
$k_{1122}=-20.4$ & $k_{1233}=7.5$ & $k_{2333}=44.3$ \\
$k_{1123}=-7.1$ & $k_{1333}=10.8$ & $k_{3333}=65.7$ \\
\hline
\end{tabular}

$50 \mathrm{ps}$ at a density of $1.1 \mathrm{~g} / \mathrm{cm}^{3}$ and a temperature of $298 \mathrm{~K}$, a long trajectory was generated from which starting configurations for nonequilibrium trajectories were sampled at $1 \mathrm{ps}$ intervals.

To excite the $\mathrm{OH}$ stretch vibration of HOD a $75 \mathrm{fs}$ Gaussian-shaped external electrical field with initial random phase oscillating at a frequency of $3400 \mathrm{~cm}^{-1}$ was applied to the HOD molecule imitating the IR laser excitation field. The pulse was interrupted when the total energy of the system had increased by $E_{\mathrm{exc}}=\hbar \omega_{\mathrm{OH}}=h c \cdot 3400 \mathrm{~cm}^{-1}$ $=40.7 \mathrm{~kJ} /$ mole. The field intensity was adjusted to reach the required energy in a $75 \mathrm{fs}$ time window around the maximum of a Gaussian pulse. Such an excitation procedure seems to be more "natural" than the instantaneous change of kinetic or/and potential energy usually employed. ${ }^{25,28}$ At the same time it ensures a random phase of the excited mode and avoids undesired excitation of other vibrational modes. ${ }^{34}$ An analogous procedure was used to selectively excite the OD stretch and the bend modes of HOD by one quantum of energy, respectively.

The computed quantities were averaged over 4800 trajectories. After excitation the individual trajectories were propagated for 25 ps. During this period the nuclear velocities and forces acting on the HOD and $\mathrm{D}_{2} \mathrm{O}$ normal coordinates were calculated following standard methods. ${ }^{48}$

\section{VER SIMULATION RESULTS}

In Fig. 1 vibrational spectra determined from dipole correlation function of $\mathrm{HOD}$ and $\mathrm{D}_{2} \mathrm{O}$ at equilibrium conditions are presented. The center of the $\mathrm{OH}$ stretch band matches the experimental value of $3400 \mathrm{~cm}^{-1}$. Also the other vibrational frequencies of $\mathrm{HOD}$ and $\mathrm{D}_{2} \mathrm{O}$ are in satisfactory agreement with measurements. It should be mentioned that the calculated librational spectra for $\mathrm{D}_{2} \mathrm{O}$ are in very good agreement with experimental ones [Fig. 1(a)], which indicates that the $\mathrm{SPC} / \mathrm{E}$ water model reproduces the rotational dynamics quite satisfactorily.

Figure 2 shows the time evolution of the excess vibrational energy in the three normal modes of HOD following OH stretch (a), OD stretch (b), and bend (c) excitation. As described in the technical part for the $\mathrm{OH}$ stretch excitation a pulsed electrical field with a $3400 \mathrm{~cm}^{-1}$ carrier frequency was applied until the total energy of the system increased by $40.7 \mathrm{~kJ} / \mathrm{mol}$, which on average took $35 \mathrm{fs}$. As demonstrated in Fig. 2(a), however, at the end of this procedure the HOD

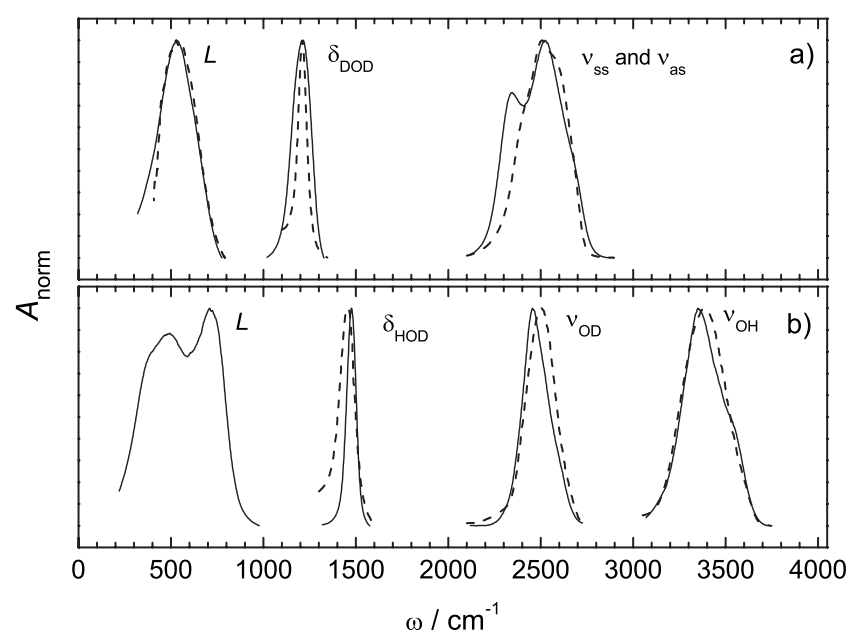

FIG. 1. Normalized spectra of $\mathrm{D}_{2} \mathrm{O}$ (a) and HOD (b). Calculated spectra (Ref. 49) (solid) are compared with experimental line shapes (Refs. 50 and 51 ) (dashed). $L$ denotes librational band (the experimental librational band of HOD is not available).

excess energy had increased to $46.5 \mathrm{~kJ} / \mathrm{mol}$. The difference of about $6 \mathrm{~kJ} / \mathrm{mol}$ turns out to be caused by the dependence of the intermolecular potential on the $\mathrm{OH}$ stretch energy, the details of which will be discussed in a subsequent article. Here it is sufficient to note that upon excitation the average $\mathrm{OH}$ distance increases due to the anharmonicity of the in-

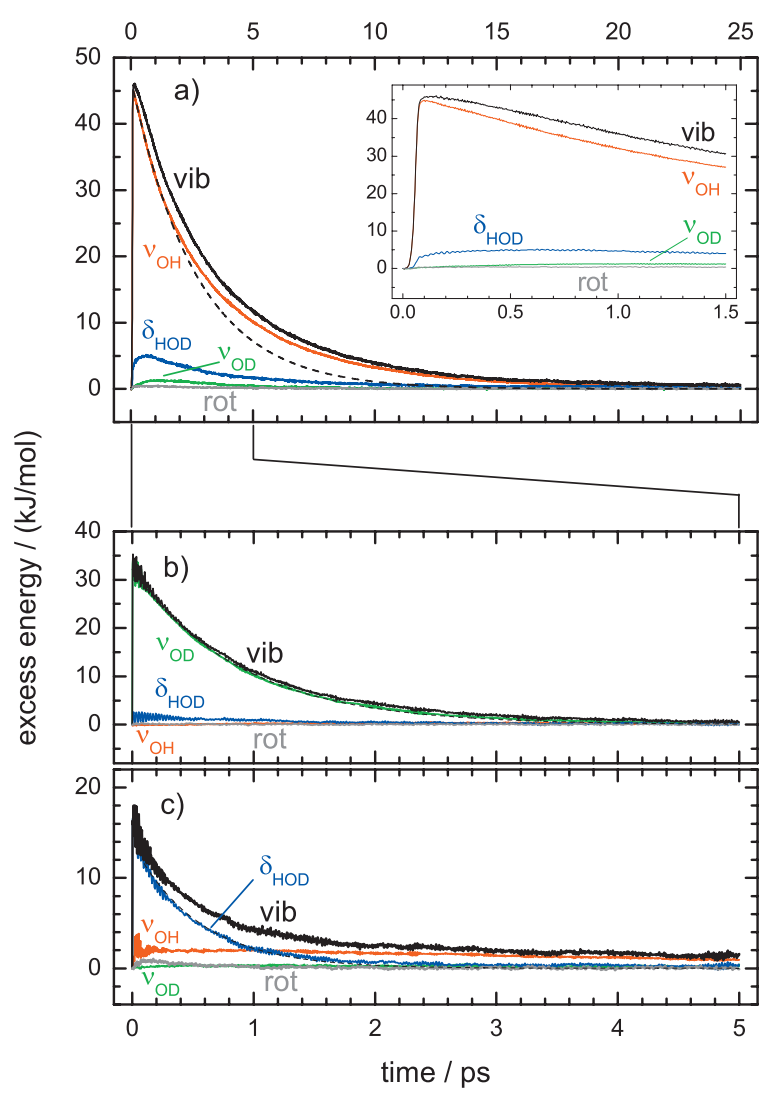

FIG. 2. (Color) Time evolution of the excess vibrational energy in HOD after exciting the $\mathrm{OH}$ stretching (a), OD stretching (b), and bending (c) vibration. Monoexponential fits to excess energy of the excited vibrations are shown as dashed lines. Note that in the case (a) the fit was applied to the initial $1.5 \mathrm{ps}$ of relaxation only. The derived time constants are $\tau_{\mathrm{OH}}$ $=2.7 \mathrm{ps}, \tau_{\mathrm{OD}}=0.90 \mathrm{ps}$, and $\tau_{\mathrm{HOD}}=0.57 \mathrm{ps}$. 
tramolecular HOD potential. The elongation of the $\mathrm{OH}$ bond induces a stronger $\mathrm{HOD}-\mathrm{D}_{2} \mathrm{O}$ interaction. During excitation a fraction of this solvation energy is directly transformed into kinetic energy of the hydrogen atom thereby increasing the $\mathrm{OH}$ stretch and bend vibrational energies of HOD by $3 \mathrm{~kJ} /$ mol each. This instantaneous response to the increased solute-solvent interaction energy is followed by solvent relaxation, i.e., rearrangement of the $\mathrm{D}_{2} \mathrm{O}$ molecules around HOD on a longer timescale. In comparison with the excitation energy the change of vibrational energy due to solvation is of minor importance. Therefore we may safely neglect its influence on the VER dynamics.

The decay of the OH-stretch vibrational energy in Fig. 2(a) can be described by biexponential function with time constants of 1.5 and $4.6 \mathrm{ps}$ and corresponding amplitudes of 17.6 and $27.7 \mathrm{~kJ} / \mathrm{mol}$. From the intramolecular potential it is evident that this biexponential shape of $\Delta E_{\mathrm{OH}}(t)$ most likely is caused by the dependence of anharmonic couplings in HOD on the excess energy. For a comparison with experiment and LT results where the relaxation rate depends on matrix elements of the excited state it is reasonable to determine the rate from the slope of the instantaneous decay directly after excitation. For this purpose an exponential function was fitted to the first 1.5 ps of the relaxation accounting for about $40 \%$ of the $\Delta E_{\mathrm{OH}}(t)$ decay, which gives a time constant of $\tau_{\mathrm{OH}}=2.7$ ps [Fig. 2(a)]. During relaxation the bending (OD stretching) energy rises within $0.5 \mathrm{ps}(1.0 \mathrm{ps})$ by $5 \mathrm{~kJ} / \mathrm{mol}(1 \mathrm{~kJ} / \mathrm{mol})$ and then relaxes similar to the total energy.

After excitation of the OD stretch and the bend the energy in these modes, to a good approximation, decays exponentially with time constants of $\tau_{\mathrm{OD}}=0.90 \mathrm{ps}$ and $\tau_{\mathrm{HOD}}$ $=0.57$ ps, as shown in Figs. 2(b) and 2(c), respectively. In both cases there are no significant concurrent energy changes in the other modes. The steplike increase in the $\mathrm{OH}$ stretch energy associated with the $\delta_{\mathrm{HOD}}$ excitation in Fig. 2(c) is caused by a similar solvation effect as it is visible in Fig. 2(a) for the $\mathrm{OH}$ excitation.

In order to reveal the VER pathways the internal and external work contributions of individual HOD degrees of freedom will be analyzed. We start with the intramolecular energy exchange of the excited $\mathrm{OH}$ vibration as presented in Fig. 3. According to Eq. (27) there are four contributions facilitating intramolecular energy flux, $W^{(\text {int })}, W^{(\mathrm{cf})}, W^{(\mathrm{cor})}$, and $W^{(\text {eul })}$. The strongest, $W^{(\text {int) }}$, is associated with anharmonic terms of the HOD intramolecular potential and leads to transfer of $21.7 \mathrm{~kJ} / \mathrm{mol}$ into the HOD bend vibration (upper panel of Fig. 3). The direct $\mathrm{OH}$ stretch to $\mathrm{OD}$ stretch channel $(0.8 \mathrm{~kJ} / \mathrm{mol})$ and nonbinary contributions $(1.2 \mathrm{~kJ} /$ mol) are of minor importance. Also coupling of the $\mathrm{OH}-$ vibration to other modes by centrifugal, Coriolis, and Euler forces is relatively weak. Similar to Fig. 3 the intramolecular energy transfer of the other modes has been calculated.

The external work done by the HOD molecule after $\mathrm{OH}$ stretch excitation is shown in Fig. 4. Notice that directly after excitation HOD gains energy from the solvent. After that the energy becomes negative and indicates energy transfer to the bath. The time dependence follows the decay of the total energy in Fig. 2. The initial positive work in Fig. 4 deserves

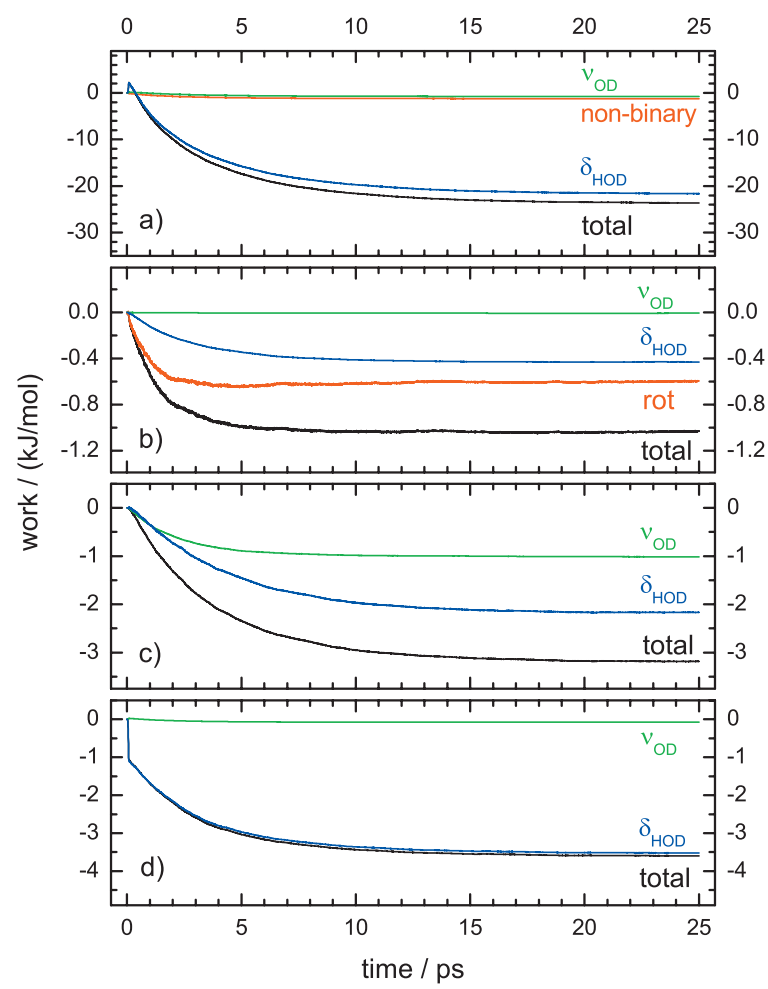

FIG. 3. (Color) Intramolecular energy exchange between the $\mathrm{OH}$ stretching vibration and other degrees of freedom caused by anharmonic couplings of the intramolecular HOD potential $W^{(\mathrm{int})}(\mathrm{a})$, as well as centrifugal $W^{(\mathrm{cf})}$ (b), Coriolis $W^{(\mathrm{cor})}(\mathrm{c})$, and Euler $W^{(\mathrm{eul})}$ (d) couplings, respectively.

further attention. Its amount is similar to the difference between the HOD vibrational energy and the total energy supplied by the excitation pulse found in Fig. 2, supporting our interpretation that it stems from an increase in the solvation energy when the HOD molecule is excited. During VER the solvation energy gradually returns to its initial value, such that the overall energy balance is not affected. Accordingly, at $25 \mathrm{ps}$ when relaxation is complete, a total work of 40.7 $\mathrm{kJ} / \mathrm{mol}$ has been performed by HOD which matches the excitation energy. The individual energy pathways determined from Fig. 4 are as follows: OH stretch, HOD bend, and OD

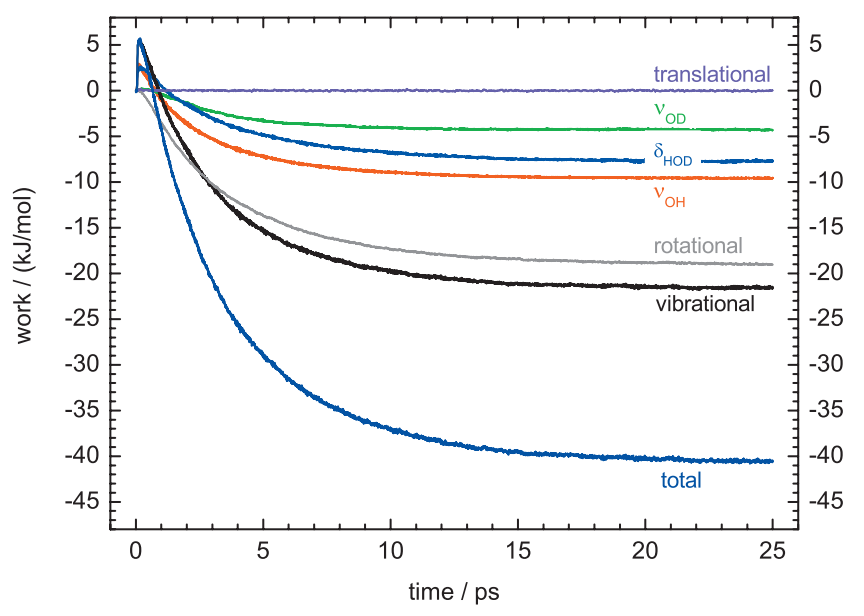

FIG. 4. (Color) Energy dissipated by the HOD degrees of freedom into the solvent. Negative sign of work here corresponds to the energy flow into the solvent. 


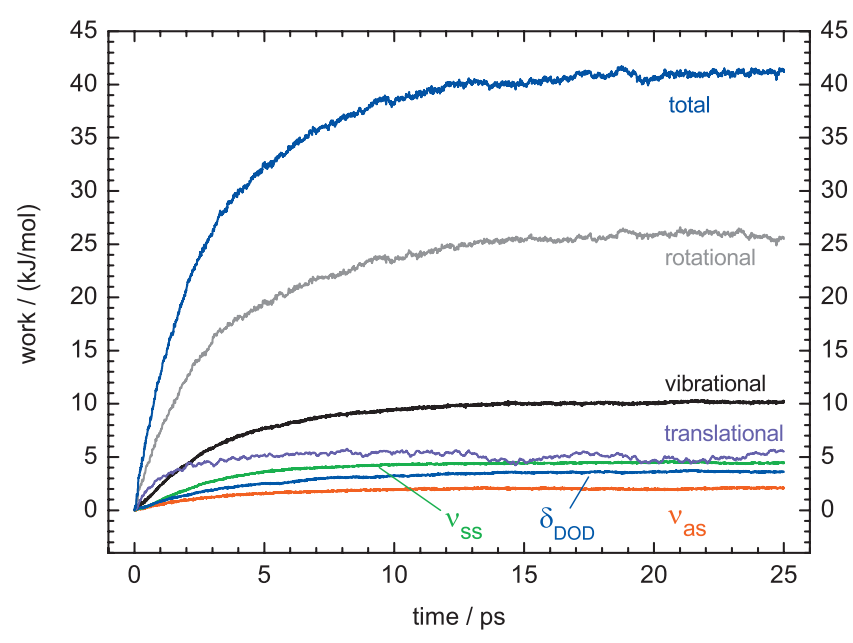

FIG. 5. (Color) Energy accepted by the solvent degrees of freedom.

stretch mode of HOD transfer $23 \%, 19 \%$, and $10 \%$ of the excess energy, respectively; $48 \%$ of the energy flows through rotational motion, whereas the translation of HOD does not contribute to energy transfer.

Complementary to these results the energy accepting modes of the $\mathrm{D}_{2} \mathrm{O}$ solvent are presented in Fig. 5. The total energy absorbed agrees with the amount delivered by HOD. The largest part, $62 \%$, excites rotational motion whereas $14 \%$ flows into the translational degrees of freedom of the $\mathrm{D}_{2} \mathrm{O}$ molecules. The remaining energy is transferred to the $\mathrm{D}_{2} \mathrm{O}$ vibrations, in which the bend receives $9 \%$ and symmetric and asymmetric stretches receive $10 \%$ and $5 \%$, respectively. Comparing Figs. 4 and 5 it is rather tempting to suppose that the $\mathrm{D}_{2} \mathrm{O}$ bend excitation results from direct V-V

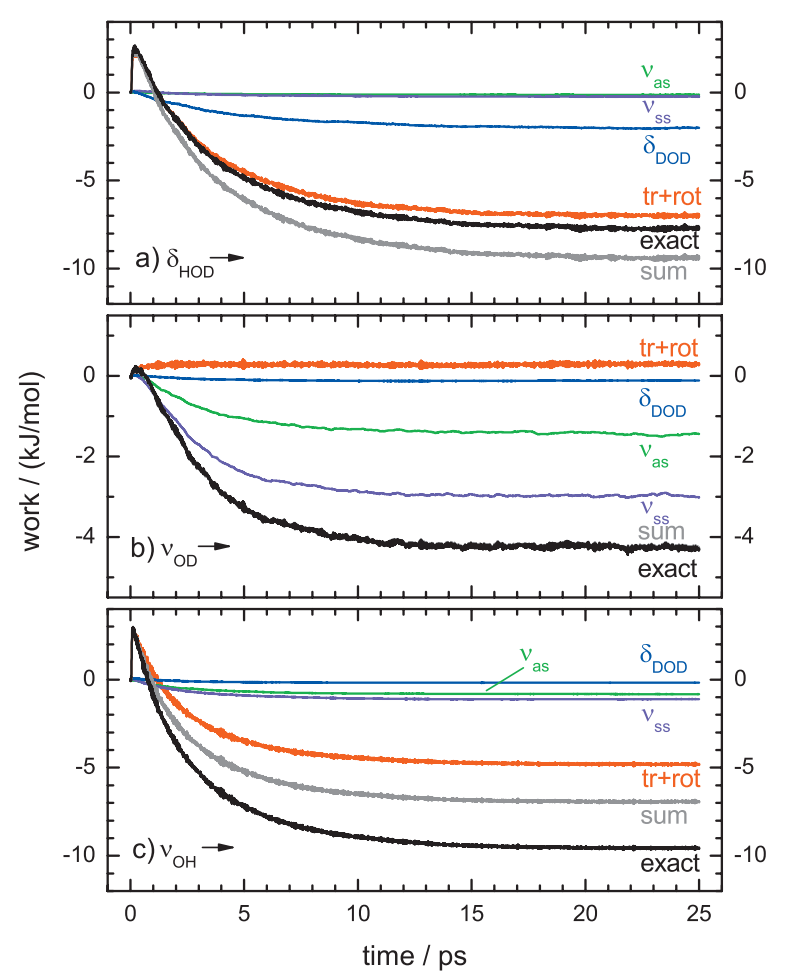

FIG. 6. (Color) Mode-to-mode energy transfer of HOD: energy release by bend (a), OD stretch (b), and $\mathrm{OH}$ stretch (c) of HOD into the $\mathrm{D}_{2} \mathrm{O}$ vibrations, respectively.

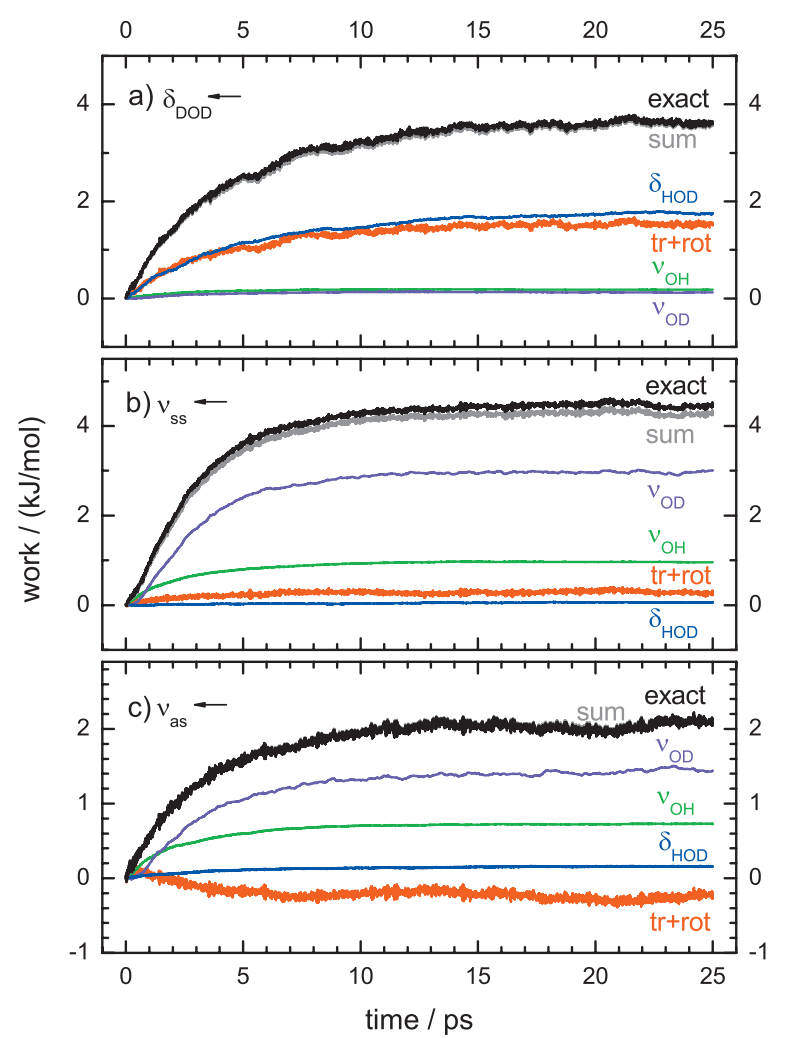

FIG. 7. (Color) Mode-to-mode energy transfer of $\mathrm{D}_{2} \mathrm{O}$ : energy accepted by bend (a), symmetric stretch (b), and asymmetric stretch (c) of $\mathrm{D}_{2} \mathrm{O}$ from the HOD vibrations.

transfer by the HOD bending mode. Similarly, the $\mathrm{D}_{2} \mathrm{O}$ stretching vibrations are most likely excited by $\mathrm{V}-\mathrm{V}$ transfer from the $\mathrm{OH}$ and $\mathrm{OD}$ stretching modes of HOD.

To prove this conjecture the mode-to-mode energy transfer was analyzed using the expansion described in Sec. II D. The results are plotted in Figs. 6 and 7, which show the VER contributions from the viewpoint of the HOD and $\mathrm{D}_{2} \mathrm{O}$ modes, respectively. As the expansion is only approximate the sum of energies released by a given HOD vibration (Fig. 6) in general does not match the exact value determined from Fig. 4. The same is true for the sum of energy contributions gained by a given $\mathrm{D}_{2} \mathrm{O}$ mode in Fig. 7 (compare Fig. 5). However, a closer look shows that the difference correlates with the degree of excitation in the mode. If it is small [Figs. 6(b) and 7] the error introduced by the expansion is negligible. Comparison of Figs. 6 and 7 suggests that deviations effect mainly V-R and V-T transfer components. On the other hand the mode-to-mode $\mathrm{V}-\mathrm{V}$ transfer components are reliable and consistently described by this method. In fact, examining the HOD and $\mathrm{D}_{2} \mathrm{O}$ bending modes [Figs. 6(a) and 7(a)] one obtains $2 \mathrm{~kJ} / \mathrm{mol}(5 \%) \mathrm{V}-\mathrm{V}$ transfer for the $\delta_{\mathrm{HOD}} \rightarrow \delta_{\mathrm{DOD}}$ channel. Figure 7(b) reveals that the entire OD stretch vibrational energy $(10 \%)$ of HOD is resonantly transferred to the $\mathrm{D}_{2} \mathrm{O}$ stretching modes. Finally, the $\mathrm{OH}$ stretching mode of HOD releases another 5\% of energy by V-V transfer to the $\mathrm{D}_{2} \mathrm{O}$ stretches.

All the VER pathways are summarized in Fig. 8 and Table II. In Fig. 8 different kinds of arrows are used to distinguish the operating coupling mechanisms. Their respective widths are proportional to the transmitted energies. The 


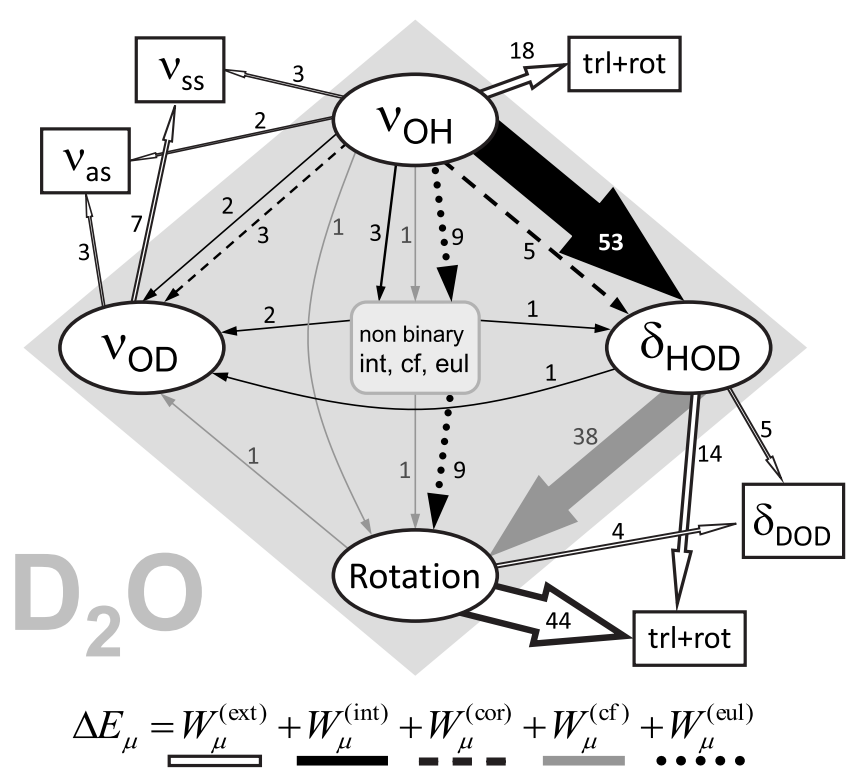

FIG. 8. Vibrational energy transfer pathways of $\mathrm{HOD}$ in $\mathrm{D}_{2} \mathrm{O}$ after exciting the $\mathrm{OH}$ stretching vibration (in $\%$, contributions $<1 \%$ have been omitted).

main relaxation channel of the $\mathrm{OH}$ stretching vibration involves energy transfer to the HOD bending vibration in which $53 \%$ is due to the binary anharmonic part $U_{2}$ of the intramolecular potential [Eq. (3)] and 5\% due to Coriolis coupling. $18 \%$ of the $\nu_{\mathrm{OH}}$ energy is directly dumped to the solvents translation and rotation and minor parts of $5 \%$ each to $\nu_{\mathrm{OD}}$ and to the $\mathrm{D}_{2} \mathrm{O}$ stretching vibrations. The remaining excess energy is transferred by nonbinary intramolecular $(3 \%)$, centrifugal $(1 \%)$, and Euler $(9 \%)$ interactions either to

TABLE II. HOD energy transfer pathways (in \%) and time constants (in ps).

\begin{tabular}{lccc}
\hline \hline Pathway $^{\mathrm{a}}$ & $\mathrm{RH}^{\mathrm{b}}$ & LS $^{\mathrm{c}}$ & This work \\
\hline \multicolumn{4}{c}{ Intramolecular } \\
$\nu_{\mathrm{OH}} \rightarrow \delta_{\mathrm{HOD}}$ & 82 & 57 & \\
$\nu_{\mathrm{OH}} \rightarrow \nu_{\mathrm{OD}}$ & 2 & 6 & 78 \\
$\nu_{\mathrm{OH}} \rightarrow$ rot & 3 & & 11 \\
$\delta_{\mathrm{HOD}} \rightarrow$ rot & 0 & & 38
\end{tabular}

\begin{tabular}{|c|c|c|c|}
\hline & nterm & & \\
\hline & & 0 & 9 \\
\hline & & 19 & 15 \\
\hline$\left(\nu_{\mathrm{ss}}+\nu_{\mathrm{as}}\right)$ & & & 62 \\
\hline $\mathrm{HOD} \rightarrow\left\{\begin{array}{c}\mathrm{ss}^{2} \text { as } \\
\text { rot }\end{array}\right.$ & & & 14 \\
\hline $\begin{array}{l}\text { rot } \\
\text { trl }\end{array}$ & & & \\
\hline l trl & & & \\
\hline$\nu_{\mathrm{OH}} \rightarrow$ bath & 16 & 38 & 23 \\
\hline$\nu_{\mathrm{OH}} \rightarrow\left(\nu_{\mathrm{ss}}+\nu_{\mathrm{as}}\right)$ & & 12 & 5 \\
\hline$\delta_{\mathrm{HOD}} \rightarrow \delta_{\mathrm{DOD}}$ & & 0 & 5 \\
\hline$\nu_{\mathrm{OD}} \rightarrow\left(\nu_{\mathrm{ss}}+\nu_{\mathrm{as}}\right)$ & & 6 & 10 \\
\hline rot $\rightarrow$ bath & & & 48 \\
\hline
\end{tabular}

\begin{tabular}{lccc}
\multicolumn{5}{c}{ Relaxation time constants } \\
$\tau_{\mathrm{OH}}$ & 7.5 & 2.3 & 2.7 \\
$\tau_{\mathrm{OD}}$ & 24 & 0.39 & 0.90 \\
$\tau_{\mathrm{HOD}}$ & 1.6 & 0.38 & 0.57 \\
\hline
\end{tabular}

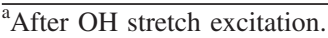

${ }^{\mathrm{b}}$ Calculated from Ref. 17.

${ }^{\mathrm{c}}$ Calculated from Ref. 18. the HOD rotation [Euler $(9 \%)$ plus centrifugal $(1 \%)]$ or to the OD stretch $(2 \%)$ and HOD bend (1\%). Most of the energy entering the bending mode transforms into HOD rotational motion by centrifugal coupling (38\%). A considerable amount of $14 \%$ of the total excess energy flows to the $\mathrm{D}_{2} \mathrm{O}$ translations and rotations. The remaining $\delta_{\mathrm{HOD}}$ energy $(5 \%)$ is delivered to the $\mathrm{D}_{2} \mathrm{O}$ bending mode by $\mathrm{V}-\mathrm{V}$ transfer. All the energy entering the $\nu_{\mathrm{OD}}$ mode is resonantly transferred to the $\mathrm{D}_{2} \mathrm{O}$ stretches.

The energy pathways derived from simulations with selective OD stretch and bend excitations are consistent with the results of Figs. 3-6, i.e., the OD stretch mode of HOD transfers $100 \%$ of its energy to the $\mathrm{D}_{2} \mathrm{O}$ stretching vibrations and for $\delta_{\mathrm{HOD}}$ we observe partioning into rotation $(53 \%)$, $\delta_{\text {DOD }}(12 \%)$ and bath rotations and translations $(32 \%)$ quite compatible with Fig. 8.

\section{DISCUSSION}

The results of our NEMD calculations can be compared with experimental findings and semiclassical calculations based on the LT theory. The experimental lifetime of the $\mathrm{OH}$ stretch vibration of $\mathrm{HOD}$ in $\mathrm{D}_{2} \mathrm{O}$ was measured to be between 0.5 and 1.0 ps. $^{4-16}$ The bend relaxation time was recently measured to be $0.39 \mathrm{ps}^{52}$ Our calculations give values of $\tau_{\mathrm{OH}}=2.7 \mathrm{ps}$ and $\tau_{\mathrm{HOD}}=0.57 \mathrm{ps}$, which is in satisfactory agreement with experiment, respectively.

The stretched exponential shape of $\Delta E_{\mathrm{OH}}(t)$ reflects the energy dependence of intramolecular couplings. It may be also possible that the averaged decay curve consists of a superposition of fast and slow decaying trajectories dependent on whether the $\mathrm{OH}$ mode at the time of excitation was embedded in a stronger or weaker hydrogen bonded environment. Just this inhomogeneous distribution of hydrogen bonded configurations is regarded to cause the broad scatter of time constants observed experimentally. We will show in a future article that both the energy dependence of intramolecular couplings and the inhomogeneity of relaxation rates contribute to the stretched exponential decay of $\Delta E_{\mathrm{OH}}(t)$.

Experimental information about the relaxation pathways stems from the work of Deak et al. ${ }^{21}$ Using an IR-pump/ Raman-probe scheme they were able to determine quantum yields for exciting the bending vibrations of $\mathrm{HOD}$ and $\mathrm{D}_{2} \mathrm{O}$ as well as the stretching modes of $\mathrm{D}_{2} \mathrm{O}$. To compare with these results our classical energy transfer efficiencies of Fig. 8 have to be translated into corresponding quantum yields. For $\delta_{\text {HOD }}$ we calculate $0.58 \cdot\left(3400 \mathrm{~cm}^{-1} / 1450 \mathrm{~cm}^{-1}\right)=1.4$ quanta, which agrees nicely with the experimentally determined minimum of 1.2 quanta of excitation. For the solvent modes we find $0.09 \cdot\left(3400 \mathrm{~cm}^{-1} / 1290 \mathrm{~cm}^{-1}\right)=0.24$ quanta of $\delta_{\mathrm{DOD}}$ and $0.15 \cdot\left(3400 \mathrm{~cm}^{-1} / 2500 \mathrm{~cm}^{-1}\right)=0.2$ quanta of OD stretch excitation, which has to be compared with $\geq 0.6$ and 0.1 , respectively. ${ }^{21}$ Owing to a Fermi resonance between the OD stretch fundamentals and the bend overtone of $\mathrm{D}_{2} \mathrm{O}$ there is efficient energy transfer from the stretches to the bend increasing the yield of $\delta_{\mathrm{DOD}}$ at the expense of $\nu_{\mathrm{SS}}$ and $\nu_{\text {as }}$ of $\mathrm{D}_{2} \mathrm{O}$. In that case better agreement with the experiment could be achieved.

The VER pathways of HOD in liquid $\mathrm{D}_{2} \mathrm{O}$ were studied 
in detail by Rey and Hynes ${ }^{17}$ and Lawrence and Skinner ${ }^{18}$ (further referred to as RH and LS, respectively) in the framework of semiclassical LT theory. Here the transition rates $k_{i f}$ from a vibrational state $i$ to a state $f$ are expressed in terms of matrix elements of the solute normal coordinates and classical TCF of the corresponding normal forces multiplied by a QCF. $^{53}$ The matrix elements were computed quantum mechanically, while the TCFs of forces were obtained from a classical MD calculation. The RH and LS approaches differ in many details, among others in the QCF whose specific form is ambiguous (see reviews ${ }^{20,54}$ for extensive comparison).

To compare with our data the LT rate constants $k_{i f}$ of $\mathrm{RH}$ and LS are used to determine the amount of energy transferred by a particular pathway, e.g., a transition from state $i$ to state $f$ has the probability $p_{i f}=k_{i f} / \Sigma_{j<i} k_{i j}$ and is associated with energy transfer to the bath of $\Delta E_{i f}=p_{i f} \hbar \omega_{i f}$. The remaining energy flows into the final state $f$. The results of these calculations are summarized in Table II. In their original paper $\mathrm{RH}$ calculated a time constant of $\tau_{\mathrm{OH}}=7.5 \mathrm{ps}$ when applying the so-called standard QCF. They identified the dominant relaxation pathway as being associated with an intramolecular transition from the excited $\mathrm{OH}$ stretch $\nu_{\mathrm{OH}}=1$ state to the bend overtone, $\delta_{\mathrm{HOD}}=2$, of HOD. In comparison with RH the theoretical treatment of LS uses different intramolecular HOD and intermolecular water potentials (TIP4P instead of SPC/E). Additionally, a more elaborate procedure with varying QCFs dependent on whether $\mathrm{V}-\mathrm{V}$ or $\mathrm{V}-\mathrm{T} / \mathrm{R}$ transfer is considered was implemented. They arrive at a notably shorter $\mathrm{OH}$ stretch lifetime of $2.3 \mathrm{ps}$ in good agreement with our calculations. However, in a recent review $\operatorname{article}^{20}$ it was demonstrated that the discrepancies between $\mathrm{RH}$ and LS are caused by improved potentials and QCFs applied in the latter work. Taking this into account the recalculated rates and pathways of RH are almost similar to those of LS. Hence for the discussion it is sufficient to compare our data only with the LS results.

Table II shows that in the LS work the $\nu_{\mathrm{OH}} \rightarrow \delta_{\mathrm{HOD}}$ channel is dominating the relaxation by transferring $57 \%$ of the energy. The intramolecular $\nu_{\mathrm{OH}} \rightarrow \nu_{\mathrm{OD}}$ pathway contributes with $6 \%$. This energy subsequently flows completely into the $\mathrm{D}_{2} \mathrm{O}$ stretching modes by $\mathrm{V}-\mathrm{V}$ transfer. The remaining $\mathrm{OH}$ stretch energy of $37 \%$ is directly transferred to the bath. All these results almost coincide with our calculations leading also to similar relaxation time constants.

On the other hand great differences become evident from Table II for the intramolecular couplings of vibrational and rotational degrees of freedom of HOD. Our results show Euler and more pronounced centrifugal couplings draining energy from the $\mathrm{OH}$ stretch $(11 \%)$ and HOD bend $(38 \%)$ vibration into the rotation of the molecule. These channels were reported to be insignificant in previous studies. ${ }^{17,18}$ The reason for this discrepancy may be that the approximations used to evaluate the centrifugal couplings in LT calculations are not justified.

Another difference concerns the energy transfer channels populating the bending mode of $\mathrm{D}_{2} \mathrm{O}$. According to our calculations the $\mathrm{V}-\mathrm{V}$ transfer channels $\delta_{\mathrm{HOD}} \rightarrow \delta_{\mathrm{DOD}}$ and rotations $\rightarrow \delta_{\mathrm{DOD}}$ are responsible for $5 \%$ and $4 \%$ of the HOD energy release, respectively. In the LS work this pathway was found to be unimportant.

\section{CONCLUSION}

The classical NEMD simulation of HOD vibrational energy relaxation in $\mathrm{D}_{2} \mathrm{O}$ reproduces the main features of previous LT calculations. ${ }^{17,18,55}$ This concerns the mean high energy $\mathrm{OH}$-stretch lifetime of $2.7 \mathrm{ps}$, the dominant participation of the intramolecular OH-stretch-to-HOD-bend channel $(58 \%)$, and a substantial amount of direct $\mathrm{OH}$ vibrational energy dissipation into bath modes (NEMD 23\%, LT 38\%). Our NEMD simulations, however, gave us the possibility to assess the extent of rotational contributions, which turned out to be quite substantial not only in providing an efficient intramolecular channel for HOD-bend depopulation (38\%), but even more so as the most dominant energy dissipation pathway into bath modes (48\%). These results suggest that the most dominant energy relaxation pathway from $\mathrm{OH}$-stretch to HOD-bend to bath passes through one additional intermediate step from HOD-bend to HOD-rotation to bath. The most effective accepting bath modes are $\mathrm{D}_{2} \mathrm{O}$ rotations $(62 \%)$ caused by the substantial spectral overlap between librational bands of $\mathrm{D}_{2} \mathrm{O}$ and $\mathrm{HOD}$ (Fig. 1). Also, there are quite a few minor channels that were identified for the first time. One of the most notable among them is transfer to the DOD-bend from the HOD-bend and HOD-rotation contributing to about $9 \%$ altogether. A comparison with experimentally determined relaxation times shows that the simulation reproduces the time scales correctly, though not too much significance should be attached to the exact values, which were obtained without any tuning of potential parameters. The measured $\mathrm{OH}$-stretch lifetime in $\mathrm{D}_{2} \mathrm{O}$ was found to be of the order of $0.5-1.0 \mathrm{ps}$, that of HOD-bend is $0.39 \mathrm{ps}$. While the former may be compared with the initial decay component $(2.7 \mathrm{ps})$ of the OH-energy relaxation in Fig. 2(a), the latter was found to be 0.57 ps [Fig. 2(c)].

Of course, our NEMD calculations comprise the complete information of the solvent response upon vibrational excitation and subsequent energy relaxation mechanism as a result of energy transfer into the intermolecular $\mathrm{DOH} \cdot \mathrm{OD}_{2}$ bond. These issues will be discussed in a future article.

\section{ACKNOWLEDGMENTS}

We thank Professor Jürgen Troe for encouraging this study. The work has been supported by the Deutsche Forschungsgemeinschaft (Grant No. SCHR 303/1-1).

\section{APPENDIX A: CORRECTION FORCES FOR ROTATIONAL INVARIANCE}

The dynamical equations of motion of the solute have to be constructed on the basis of intramolecular normal forces $F_{\mu}$ that are easily calculated by differentiating the potential energy [Eq. (38)] with respect to the normal coordinates. The intramolecular atomic forces are calculated by the linear transformations ${ }^{41}$ 


$$
\boldsymbol{F}_{a}=\sqrt{m_{a}} \sum_{\mu} \boldsymbol{L}_{a \mu} F_{\mu}
$$

When the Eckart frame is used these forces must be applied to the equilibrium positions of atoms to preserve rotational invariance. However, the equations of motion that are numerically integrated contain the actual positions of atoms and the compensation contributions have to be calculated to restore the rotational invariance. These contributions must not disturb the evolution of the normal coordinates but solely have to correct the rotational motion of the molecule.

The intramolecular forces being applied at the actual atomic positions result in nonzero total torque

$$
\boldsymbol{M} \equiv \boldsymbol{M}_{C}^{(i)}=\sum_{a, \mu} \sqrt{m_{a}} \boldsymbol{r}_{a} \times \boldsymbol{L}_{a \mu} F_{\mu} .
$$

The compensation forces $\delta \boldsymbol{F}_{a}$ must be linear in $\boldsymbol{M}$ and in $m_{a} \boldsymbol{r}_{a}$. The latter requirement is necessary to keep the sum of these forces over the solute atoms at zero. As the torque is a pseudovector, the corresponding relation is as follows:

$$
\delta F_{a j}=m_{a} \epsilon_{j l m} r_{a l} S_{m n} M_{n},
$$

where the summation convention is implied and $\epsilon_{j l m}$ is fully antisymmetric Levi-Civita symbol. ${ }^{41}$

The unknown tensor $S$ is determined by the condition that the compensation forces applied to the actual atomic positions correct the torque to zero,

$$
\sum_{a} \epsilon_{i k j} r_{a k} \delta F_{a j}=-M_{i}
$$

Substituting Eq. (A3) in Eq. (A4) and remembering that

$$
\epsilon_{i k j} \epsilon_{j l m}=\delta_{i l} \delta_{k m}-\delta_{i m} \delta_{k l},
$$

the following equation is obtained:

$$
\left(\delta_{i l} \delta_{k m}-\delta_{i m} \delta_{k l}\right) R_{k l} S_{m n}=-\delta_{i n},
$$

where

$$
R_{k l}=\sum_{a} m_{a} r_{a k} r_{a l}
$$

Equation (A6) can be rewritten as

$$
\boldsymbol{I} \cdot \boldsymbol{S}=\boldsymbol{E},
$$

where

$$
\boldsymbol{I}=(\operatorname{Tr} \boldsymbol{R}) \boldsymbol{E}-\boldsymbol{R}
$$

is the inertia tensor. Thus,

$$
S=I^{-1} \text {. }
$$

Equations (A3) and (A10) define the correction forces that have to be added to the calculated forces $\boldsymbol{F}_{a}$. It is easy to show that the projections of the full system of the correction forces on the normal coordinates are all equal to zero.

$a, b$, and $c$ are the principal values of the tensor $\boldsymbol{I}$. Then the principal values of the tensor $S$ are $1 / a, 1 / b$ and $1 / c$ in the same system of principal axes. For a planar molecule the vector $\boldsymbol{M}$ is orthogonal to the molecular plane and Eq. (A3) reduces to

$$
\delta \boldsymbol{F}_{a}=\left(m_{a} / c\right) \boldsymbol{r}_{a} \times \boldsymbol{M},
$$

where

$$
c=\sum_{a} m_{a} r_{a}^{2}
$$

In accordance with Eq. (A11) the correction forces are orthogonal to the atomic position vectors in the center of mass reference system and lie in the molecular plane.

\section{APPENDIX B: VIBRATIONAL-ROTATIONAL COUPLING TERMS}

To identify the coupling between vibrational and rotational motion $^{37}$ [Eq. (25)] one can consider time derivatives of the rotational kinetic energy [see Eq. (4)]

$$
\begin{aligned}
\frac{d K_{\mathrm{rot}}}{d t} & =\frac{d}{d t}\left(\frac{1}{2} \boldsymbol{\omega} \cdot \boldsymbol{I} \cdot \boldsymbol{\omega}\right) \\
& =\boldsymbol{M}_{C} \cdot \boldsymbol{\omega}-\frac{1}{2} \boldsymbol{\omega} \cdot \dot{\boldsymbol{I}} \cdot \boldsymbol{\omega}-\boldsymbol{\omega} \cdot \sum_{\mu \nu} \zeta_{\mu \nu} Q_{\mu} \ddot{Q}_{\nu},
\end{aligned}
$$

the Coriolis energy

$$
\begin{aligned}
\frac{d K_{\mathrm{cor}}}{d t} & =\frac{d}{d t}\left(\boldsymbol{\omega} \cdot \sum_{\mu \nu} \zeta_{\mu \nu} Q_{\mu} \dot{Q}_{\nu}\right) \\
& =\boldsymbol{\omega} \cdot \sum_{\mu \nu} \zeta_{\mu \nu} Q_{\mu} \ddot{Q}_{\nu}-\dot{\boldsymbol{\omega}} \cdot \sum_{\mu \nu} \zeta_{\mu \nu} Q_{\mu} \dot{Q}_{\nu},
\end{aligned}
$$

and the vibrational energy

$$
\frac{d E_{\mathrm{vibr}}}{d t}=\sum_{\mu} F_{\mu}^{(e)} \dot{Q}_{\mu}+\frac{1}{2} \boldsymbol{\omega} \cdot \dot{\boldsymbol{I}} \cdot \boldsymbol{\omega}+\dot{\boldsymbol{\omega}} \cdot \sum_{\mu \nu} \zeta_{\mu \nu} Q_{\mu} \dot{Q}_{\nu} .
$$

Comparison with the last term in Eqs. (B2) and (B3), respectively, reveals that the last term in Eq. (25) describes the energy exchange of vibrational mode $\mu$ with the Coriolis energy. This exchange is governed by the angular acceleration $\dot{\boldsymbol{\omega}}$ and is named Euler's contribution.

The term $(1 / 2) \boldsymbol{\omega} \cdot \dot{\boldsymbol{I}} \cdot \boldsymbol{\omega}$ in Eqs. (B1) and (B3) describes energy exchange between vibrations and rotations due to centrifugal coupling. The remaining term [last one on the right hand side of Eq. (B1) and the first one of Eq. (B2)] describes the energy exchange between rotational motion and the Coriolis energy not entering Eq. (25).

The sum of Eqs. (B1)-(B3),

$$
\frac{d}{d t}\left(E_{\mathrm{vibr}}+K_{\mathrm{rot}}+K_{\mathrm{cor}}\right)=\sum_{\mu} F_{\mu}^{(e)} \dot{Q}_{\mu}+\boldsymbol{M}_{C} \cdot \boldsymbol{\omega},
$$

shows that the energy flux from the excited solute to the bath occurs finally via direct energy exchange of vibrations and rotations with the bath, respectively. All the other terms considered above contribute to intramolecular energy redistribution.

\footnotetext{
${ }^{1}$ Ultrafast Infrared and Raman Spectroscopy, edited by M. D. Fayer (Marcel Dekker, New York, 2001).

${ }^{2}$ Ultrafast Hydrogen Bonding Dynamics and Proton Transfer Processes in the Condensed Phase, edited by T. Elsaesser and H. J. Bakker (Kluwer, Dordrecht, 2002).

${ }^{3}$ E. T. J. Nibbering and T. Elsaesser, Chem. Rev. (Washington, D.C.) 104,
} 
1887 (2004).

${ }^{4}$ S. Bratos, G. M. Gale, G. Gallot, F. Hache, N. Lascoux, and J.-C. Leicknam, Phys. Rev. E 61, 5211 (2000).

${ }^{5}$ G. M. Gale, G. Gallot, F. Hache, N. Lascoux, S. Bratos, and J.-C. Leicknam, Phys. Rev. Lett. 82, 5211 (1999).

${ }^{6}$ A. J. Lock and H. J. Bakker, J. Chem. Phys. 117, 1708 (2002).

${ }^{7}$ S. Woutersen, U. Emmerichs, and H. J. Bakker, Science 278, 658 (1997).

${ }^{8}$ S. Woutersen, U. Emmerichs, H.-K. Nienhuys, and H. J. Bakker, Phys. Rev. Lett. 81, 1106 (1998).

${ }^{9}$ G. M. Gale, G. Gallot, and N. Lascoux, Chem. Phys. Lett. 311, 123 (1999).

${ }^{10}$ R. Laenen, C. Rauscher, and A. Laubereau, Phys. Rev. Lett. 80, 2622 (1998).

${ }^{11}$ R. Laenen, C. Rauscher, and A. Laubereau, J. Phys. Chem. B 102, 9304 (1998)

${ }^{12}$ H.-K. Nienhuys, S. Woutersen, R. A. van Santen, and H. J. Bakker, J. Chem. Phys. 111, 1494 (1999).

${ }^{13}$ Z. Wang, A. Pakoulev, Y. Pang, and D. D. Dlott, J. Phys. Chem. A 108, 9054 (2004)

${ }^{14}$ Z. Wang, Y. Pang, and D. D. Dlott, Chem. Phys. Lett. 397, 40 (2004).

${ }^{15}$ D. Schwarzer, J. Lindner, and P. Vöhringer, J. Chem. Phys. 123, 161105 (2005).

${ }^{16}$ D. Schwarzer, J. Lindner, and P. Vöhringer, J. Phys. Chem. A 110, 2858 (2006).

${ }^{17}$ R. Rey and J. T. Hynes, J. Chem. Phys. 104, 2356 (1996)

${ }^{18}$ C. P. Lawrence and J. L. Skinner, J. Chem. Phys. 119, 1623 (2003).

${ }^{19}$ C. P. Lawrence and J. L. Skinner, J. Chem. Phys. 119, 3840 (2003).

${ }^{20}$ R. Rey, K. B. Möller, and J. T. Hynes, Chem. Rev. (Washington, D.C.) 104, 1915 (2004).

${ }^{21}$ J. C. Deak, S. T. Rhea, L. K. Iwaki, and D. D. Dlott, J. Phys. Chem. A 104, 4866 (2000).

${ }^{22}$ A. Staib and J. T. Hynes, Chem. Phys. Lett. 204, 197 (1993).

${ }^{23}$ C. P. Lawrence and J. L. Skinner, J. Chem. Phys. 117, 5827 (2002).

${ }^{24}$ J. R. Brown, C. B. Harris, and J. C. Tully, J. Chem. Phys. 89, 6687 (1988).

${ }^{25}$ R. M. Whitnell, K. R. Wilson, and J. T. Hynes, J. Chem. Phys. 96, 5354 (1992).

${ }^{26}$ I. Chorny, J. Vieceli, and I. Benjamin, J. Chem. Phys. 116, 8904 (2002).

${ }^{27}$ C. Heidelbach, I. I. Fedchenia, D. Schwarzer, and J. Schroeder, J. Chem. Phys. 108, 10152 (1998)

${ }^{28}$ C. Heidelbach, V. S. Vikhrenko, D. Schwarzer, and J. Schroeder, J. Chem. Phys. 110, 5286 (1999).

${ }^{29}$ G. Käb, C. Schröder, and D. Schwarzer, Phys. Chem. Chem. Phys. 4, 271 (2002).

${ }^{30}$ V. N. Kabadi and B. M. Rice, J. Phys. Chem. A 108, 532 (2004).

${ }^{31}$ A. Kandratsenka, J. Schroeder, D. Schwarzer, and V. S. Vikhrenko, Phys.
Chem. Chem. Phys. 7, 1205 (2005).

${ }^{32}$ A. Kandratsenka, J. Schroeder, D. Schwarzer, and V. S. Vikhrenko, Phys. Chem. Chem. Phys. 9, 1688 (2007).

${ }^{33}$ D. Schwarzer, P. Kutne, C. Schröder, and J. Troe, J. Chem. Phys. 121, 1754 (2004).

${ }^{34}$ S. G. Ramesh and E. L. Sibert III, J. Chem. Phys. 125, 244513 (2006).

${ }^{35}$ C. Heidelbach, J. Schroeder, D. Schwarzer, and V. S. Vikhrenko, Chem. Phys. Lett. 291, 333 (1998).

${ }^{36}$ J. K. G. Watson, Mol. Phys. 15, 479 (1968).

${ }^{37}$ E. B. Wilson, Jr., J. C. Decius, and P. C. Cross, Molecular Vibrations: The Theory of Infrared and Raman Vibrational Spectra (McGraw-Hill, New York, 1955).

${ }^{38}$ P. R. Bunker, Molecular Symmetry and Spectroscopy (Academic, New York, 1979).

${ }^{39}$ C. Eckart, Phys. Rev. 47, 552 (1935).

${ }^{40}$ G. Amat and L. Henry, Cah. Phys. 12, 273 (1958).

${ }^{41}$ H. Goldstein, Classical Mechanics (Addison-Wesley, Cambridge, MA, 1950).

${ }^{42}$ C. Lanczos, The Variational Principles of Mechanics, 4th ed. (Dover, New York, 1986).

${ }^{43}$ M. P. Allen and D. J. Tildesley, Computer Simulation of Liquids (Clarendon, Oxford, 1989).

${ }^{44}$ C. P. Lawrence and J. L. Skinner, Chem. Phys. Lett. 372, 842 (2003).

${ }^{45}$ M. G. Sceats and S. A. Rice, J. Chem. Phys. 71, 973 (1979).

${ }^{46}$ K. Kushitsu and Y. Morino, Bull. Chem. Soc. Jpn. 38, 814 (1965).

${ }^{47}$ K. Refson, Comput. Phys. Commun. 126, 310 (2000).

${ }^{48}$ J. T. Yardley, Introduction to Molecular Energy Transfer (Academic, New York, 1980)

${ }^{49}$ Neglecting the frequency dependence of the refractive index the IR absorption in the classical limit is proportional to $\omega^{2} \int d t e^{-i \omega}\langle\boldsymbol{\mu}(t) \cdot \boldsymbol{\mu}(0)\rangle$, where $\boldsymbol{\mu}$ is the dipole moment of the molecule (Ref. 56).

${ }^{50}$ M. Falk and T. A. Ford, Can. J. Chem. 44, 1699 (1966).

${ }^{51}$ The librational part of the $\mathrm{D}_{2} \mathrm{O}$ spectrum was measured in a Fourier transform infrared spectrometer.

${ }^{52}$ P. Bodis, O. F. A. Larsen, and S. Woutersen, J. Phys. Chem. A 109, 5303 (2005).

${ }^{53}$ D. W. Oxtoby, Adv. Chem. Phys. 47, 487 (1981).

${ }^{54}$ S. A. Egorov, K. F. Everitt, and J. L. Skinner, J. Phys. Chem. A 103, 9494 (1999).

${ }^{55}$ As noted in Sec. V, Refs. 17 and 18 find an important initial energy transfer from the $\mathrm{OH}$ stretch to the overtone of the HOD bend. Unfortunately, the pathway analysis with classical NEMD does not give us the possibility to consider overtones as separate energy stocks. Therefore, we only refer to the energy flow from one mode to another.

${ }^{56}$ R. Zwanzig, Nonequilibrium Statistical Mechanics (Oxford University Press, New York, 2001). 